\title{
Clinical Pearl Commentary
}

\section{By Lawrence H. Phillips, II, M.D.}

\section{Professor of Neurology, University of Virginia}

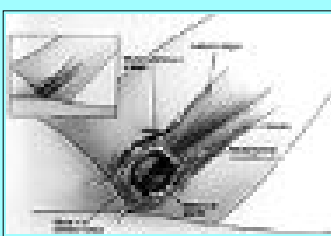

Peripheral nerve biopsy for selected indications in neuromuscular diseases can be very useful in arriving at a diagnosis. The gold standard for nerve biopsy has been the sural nerve, but its usefulness is limited in disorders that are primarily motor or those that affect the proximal nerves. The lack of a suitable motor nerve biopsy has hampered the precise pathological definition of motor neuropathies such as multifocal motor neuropathy with conduction block. This report of a technique for biopsy of a proximal motor nerve is a welcome addition to our diagnostic armamentarium.

I am concerned, however, about the sensitivity of gracilis motor nerve biopsy. In most of the disorders the authors list as possible indications for biopsy, weakness of thigh adduction is not often a prominent part of the disease. This may indicate that this proximally innervated site is relatively spared. I presume that data on the yield of biopsy at this site are contained in the authors' yet-to-be published paper in Muscle and Nerve (their reference 2).

I would reserve biopsy of this site for patients who clearly have proximal innervation involvement and for whom sural nerve biopsy is not indicated or not possible. Proximal involvement can be demonstrated by the clinical findings of weakness and sensory loss in the appropriate areas or by electromyographic findings of denervation in appropriate muscles, including the gracilis.

$\underline{\text { Click here to return to Clinical Pearl. }}$

Click here to comment: jneuro@ virginia.edu 